\title{
3 Zeitliche Restriktionen als hochschulexterne Bedingungsfaktoren struktureller Studierbarkeit
}

Dieses Kapitel fokussiert die Lebens- und Studiensituation, den Studienerfolg sowie die zeitlichen Restriktionen von vier unterschiedlichen Studierendengruppen: erwerbstätigen Studierenden, Studierenden mit Kind, Studierenden mit Pflegeaufgaben und behinderten Studierenden. Diese Analysen sind relevant, um das im Modell der strukturellen Studierbarkeit enthaltene Konzept der zeitlichen Restriktionen entwickeln zu können (siehe Abschnitt 3.5). Denn im Modell gehen nicht die einzelnen Studierendengruppen in die Analysen ein, sondern die Gemeinsamkeit der zeitlichen Restriktion.

Die Forschung zu den vier Studierendengruppen ist umfangreich. Tabelle 2 bietet zur Orientierung einen ersten Überblick über den Forschungsstand.

Tabelle 2 Übersicht über den Forschungsstand zu nicht-traditionellen Studierendengruppen.

\begin{tabular}{lcccc}
\hline Thema & $\begin{array}{c}\text { Studierende mit... } \\
\text { Erwerbs- } \\
\text { tätigkeit }\end{array}$ & Kind & $\begin{array}{c}\text { Pflege- } \\
\text { aufgaben }\end{array}$ & $\begin{array}{c}\text { Behinderun } \\
\text { angation }\end{array}$ \\
Vereinbarkeit & - & - & - & - \\
\hline Soziale Integration & $\mathrm{o}$ & - & - & - \\
\hline Akad. Integration & $\mathrm{o}$ & $\mathrm{o}$ & $/$ & $\mathrm{o}$ \\
\hline Kompetenzerwerb & + & + & $/$ & $/$ \\
\hline Studiendauer & - & - & $/$ & - \\
\hline Studienabbruch & $\mathrm{o}$ & $\mathrm{o}$ & $\mathrm{o}$ & - \\
\hline $\begin{array}{l}-=\text { negativer Effekt, }+= \\
\text { keine Informationen. }\end{array}$ & &
\end{tabular}

\subsection{Erwerbstätige Studierende}

Die Situation erwerbstätiger Studierender wird nachfolgend in vier Unterkapiteln dargestellt. Zunächst steht die Lebens- und Studiensituation im Fokus, gefolgt vom Studierverhalten und dem Studienerfolg. Das Kapitel schließt mit einer Analyse der Anforderungen von erwerbstätigen Studierenden an das Studium. 


\subsubsection{Lebens- und Studiensituation}

68 Prozent der Studierenden an deutschen Hochschulen arbeiten neben dem Studium, wobei der Anteil an Universitäten etwas höher ist als an Fachhochschulen. Viele Studierende arbeiten nicht ununterbrochen, sondern nur in den Semesterferien oder für kürzere Zeit. Insgesamt sind 38 Prozent der Studierenden laufend und während des ganzen Jahres erwerbstätig. Besonders im postgradualen Studium arbeiten die Studierenden durchgehend. In jüngster Zeit ist der Anteil der Erwerbstätigen gestiegen, so von 2012 bis 2016 um sechs Prozentpunkte (Middendorff et al. 2017, S. 60 f.).

Tabelle 3 Erwerbstätige Studierende in Deutschland. Eigene Darstellung mit Daten aus Middendorff et al. 2013, 2017.

\begin{tabular}{|c|c|c|}
\hline \multicolumn{3}{|c|}{ Erwerbstätigkeit im Studium } \\
\hline Gesamt & & $68 \%$ \\
\hline \multirow[t]{2}{*}{ Hochschultyp } & Fachhochschule & $65 \%$ \\
\hline & Universität & $69 \%$ \\
\hline \multirow{2}{*}{$\begin{array}{l}\text { Durchschnittlicher } \\
\text { Umfang pro Woche* }\end{array}$} & Erststudium & 13 Stunden \\
\hline & Postgraduales Studium & 26 Stunden \\
\hline \multirow[t]{2}{*}{ Alter* } & 20 Jahre & $41 \%$ \\
\hline & 25 Jahre & $70 \%$ \\
\hline \multirow[t]{2}{*}{ Geschlecht } & Frauen & $70 \%$ \\
\hline & Männer & $66 \%$ \\
\hline \multirow[t]{2}{*}{ Bildungshintergrund } & Niedrig & $69 \%$ \\
\hline & Hoch & $64 \%$ \\
\hline \multirow{7}{*}{$\begin{array}{l}\text { Gründe } \\
\text { 5er-Skala „trifft voll } \\
\text { und ganz zu“ } \\
\text { Mehrfachnennungen } \\
\text { möglich }\end{array}$} & Sich mehr leisten können & $38 \%$ \\
\hline & Notwendig für den & $43 \%$ \\
\hline & Lebensunterhalt & \\
\hline & Unabhängigkeit von den Eltern & $34 \%$ \\
\hline & Sammlung praktischer & $28 \%$ \\
\hline & Erfahrungen & \\
\hline & Kontakte für spätere & $17 \%$ \\
\hline
\end{tabular}

* Zeitpunkt 2012. Alle Andere Daten: 2016

Wie oft und in welchem Umfang Studierende einer Beschäftigung nachgehen, hängt von mehreren Faktoren ab. Zunächst sind dies strukturelle, regionale Faktoren. In einigen Städten, insbesondere in Westdeutschland, sind die Lebenshaltungskosten besonders hoch. In diesen Städten arbeiten die 
Studierenden deutlich häufiger (Middendorff et al. 2017, S. 61). Ein weiterer Faktor ist die Arbeitslosenquote, da der Arbeitsmarkt bei geringer Arbeitslosigkeit gute Beschäftigungsmöglichkeiten für Studierende bereithält (Häkkinen 2006, S. 168). $\mathrm{Zu}$ den strukturellen Faktoren kommen noch die individuellen Interessen und finanziellen Bedarfe hinzu. Studierende arbeiten primär, um sich mehr leisten zu können (42 Prozent) und damit ihren Anforderungen an den Lebensstandard zu erfüllen oder um sich ihren Lebensunterhalt zu verdienen (40 Prozent). Weitere wichtige Gründe hängen mit den Berufschancen zusammen, da sie sich durch praktische Erfahrungen (26 Prozent) und Kontakte in den Arbeitsmarkt (15 Prozent) bessere Chancen erhoffen (Beerkens et al. 2011, S. 686; Berthold und Leichsenring o. J.b, S. 12; Hall 2010, S. 445; Middendorff et al. 2013, S. 375). Die genannten Gründe unterscheiden sich je nach dem Bildungshintergrund der Studierenden. Denn obwohl Studierende mit hohem Bildungshintergrund fast so häufig arbeiten wie Studierende mit niedrigem Bildungshintergrund, ist für zweite Gruppe die Sicherung des Lebensunterhaltes fast doppelt so wichtig (Middendorff et al. 2017, S. 62 f.). Studierende sind damit unabhängig von ihrem sozioökonomischen Hintergrund ähnlich häufig erwerbstätig, die Gründe hierfür differieren allerdings. Dieser Befund gilt auch für andere Länder, etwa Österreich und Großbritannien (Brandstätter und Farthofer 2003, S. 137; Callender 2008, S. 368). Schließlich sind hohe Studienbelastungen oder private Lebenssituationen durch Pflegeverantwortung oder Kinder Gründe, keine Erwerbstätigkeit aufzunehmen. Die Gründe der Sorgeaufgaben treffen besonders auf Mütter zu, da nur sie prozentual seltener erwerbstätig sind (Middendorff et al. 2017, S. 62).

Die Erwerbstätigkeit umfasst im Erststudium durchschnittlich 13 Stunden pro Woche und im postgradualen Studium 26 Stunden pro Woche. Damit steigt die Erwerbstätigkeit in den höheren Semestern und mit höherem Alter deutlich an. Von den 20-Jährigen arbeiten nur 41 Prozent, wohingegen es bei den 25-Jährigen schon 70 Prozent sind. Studierende, die ihr Studium wechseln oder unterbrechen, sind um etwa 10 Prozentpunkte häufiger erwerbstätig (Middendorff et al. 2013, S. 378).

Die Terminologie in der Forschung zu arbeitenden Studierenden nutzt die Begriffe Berufs- und Erwerbstätigkeit. Eine Erwerbstätigkeit liegt dabei immer vor, wenn Studierende gegen ein Entgelt arbeiten; Berufstätigkeit fokussiert dagegen stärker die Ausübung eines erlernten Berufs.

\subsubsection{Studierverhalten}

Eine Erwerbstätigkeit kann sich aufgrund der dafür aufzuwendenden Zeit und Energie einschränkend auf das Studierverhalten auswirken. Die individuelle Gestaltung des Lernens manifestiert sich im Besuch von Lehrveranstaltungen, im 
Selbststudium und im Ablegen von Prüfungen (siehe dazu auch Kapitel 5). $\mathrm{Ob}$ Studierende beispielsweise Schwierigkeiten haben, an Lehrveranstaltungen teilzunehmen, hängt von der Vereinbarkeit von Berufstätigkeit und Studium ab. Der Begriff der Vereinbarkeit ist dabei nicht unproblematisch. Preuß (2014, S. 150) kritisiert, dass Vereinbarkeit ein konfliktfreies Zusammenbringen von unterschiedlichen Lebenswelten suggeriere. Dies ist jedoch keinesfalls bei allen berufstätigen Studierenden der Fall, da etwa die Hälfte der Studierenden von Stress und schwierigen Aushandlungen zwischen Arbeitsstelle, Familie, Freunden und Studium berichten (z.B. Broadbridge und Swanson 2006; Kember 1999). Die Frage nach der Vereinbarkeit von Studium und Erwerbstätigkeit steht im Mittelpunkt dieses Unterkapitels.

Zunächst ist zu konstatieren, dass Erwerbstätigkeit nicht per se die Gestaltung des Studiums und des Lernens einschränkt. So geben 44 Prozent der Befragten einer australischen Studie (Hall 2010, S. 444) und 30 Prozent der Studierenden einer österreichischen Studie (Pechar und Wroblewski 1998, S. 100) an, keine Vereinbarkeitsprobleme zu spüren. Die Probleme durch Erwerbstätigkeit entstehen im Zusammenspiel zwischen den individuellen Anforderungen der Arbeitsstelle, dem Umfang der Erwerbstätigkeit, der Gestaltung der Studienstrukturen und den Einstellungen der Lehrenden. Die beiden letzten Aspekte differieren je nach Studiengang oder Studienrichtung, wie Pechar und Wroblewski (1998, S. 105) zeigen.

Berichten Studierende von einer schlechten Vereinbarkeit, stellen die Zeitknappheit und die Koordination von beruflichen, studentischen und privaten Terminen die größten Herausforderungen dar. Etwa die Hälfte der Studierenden gibt an, nicht ausreichend Zeit für den Besuch von Lehrveranstaltungen, die Vorund Nachbereitung von Lehrveranstaltungen sowie die Prüfungsvorbereitung zu haben (Berthold und Leichsenring o. J.b, S. 10; Schlögl und Neubauer 2006, S. 50). Mindestens 60 Prozent der erwerbstätigen Studierenden klagen über zu wenig Freizeit und Zeit für die Familie (Broadbridge und Swanson 2006, S. 169; Pechar und Wroblewski 1998, S. 104; Schlögl und Neubauer 2006, S. 50). Anwesenheitspflichten und für die jeweilige berufliche Tätigkeit unpassende Veranstaltungszeiten sowie Überschneidungen von Lehrveranstaltungen erhöhen die Schwierigkeiten der Vereinbarkeit. Wenn Studierende ebenfalls familiäre Verpflichtungen aufweisen, verstärkt sich diese Problematik (Hall 2010, S. 444; Hawkins et al. 2005, S. 17; Nienhüser et al. 2000, S. 33; Pechar und Wroblewski 1998, S. 104). Neben familiären Verpflichtungen spielt auch die Art und die Flexibilität der Erwerbstätigkeit eine Rolle. Studierende mit studiennaher Tätigkeit haben etwas seltener Probleme, an Lehrveranstaltungen teilzunehmen (38 Prozent versus 45 Prozent) (Berthold und Leichsenring o. J.b, S. 18). Ein Grund hierfür ist die hohe zeitliche Flexibilität der Arbeitszeiten innerhalb der 
Hochschulen, die einen Teil der studiennahen Tätigkeiten ausmachen. Auch der Umfang der Erwerbstätigkeit beeinflusst die Vereinbarkeit. Je höher die wöchentliche Arbeitszeit, desto größer sind die Vereinbarkeitsprobleme. Terminprobleme bei Lehrveranstaltungen sind in diesem Sinne für etwa die Hälfte der Teilzeitberufstätigen und 87 Prozent der Vollzeitberufstätigen relevant (Pechar und Wroblewski 1998, S. 101). Auch die Sorge, das Studium nicht zu schaffen, oder die Aufregung vor Prüfungen steigen, wenn Studierende in größerem Umfang berufstätig sind (Nienhüser et al. 2000, S. 34). Arbeiten Studierende, sind sie häufiger gestresst und haben den Eindruck, sich gut organisieren zu müssen (Broadbridge und Swanson 2006, S. 175; Dundes und Marx 2006, S. 114; Evans et al. 2014, S. 85; Moreau und Leathwood 2006, S. 25). Eine qualitative Studie aus dem Vereinigten Königreich zeigt, dass erwerbstätige Studierende immer wieder neue Strategien suchen, um Studium und Beruf zu vereinbaren. Sie sehen dabei die Verantwortung überwiegend bei sich selbst und stellen wenige Anforderungen an die Hochschule (Moreau und Leathwood 2006, S. 32). Ermöglichen die Studienstruktur und der Studienverlaufsplan allerdings Freiräume für Erwerbstätigkeit, so steigt die Chance auf eine gute Einschätzung der Vereinbarkeit stark an (Mertens 2013, S. 47).

Mehrere Studien untersuchen, ob ein hoher Stresslevel aufgrund schlechter Vereinbarkeit von Studium und Beruf zu einem schlechten Gesundheitszustand der Studierenden führt. Diese Annahme kann jedoch nicht eindeutig belegt werden. Der Umfang der Erwerbstätigkeit hat keinen Einfluss auf die generelle gesundheitliche Belastung der Studierenden (Miller et al. 2008, S. 678). Vollzeitberufstätige Studierende sind ähnlich stark gesundheitlich belastet wie Studierende, die nicht arbeiten (Pechar und Wroblewski 1998, S. 110).

Der Lernprozess wird nicht nur von der Vereinbarkeit, sondern auch von der Motivation und den Kompetenzen der Studierenden beeinflusst. In diesem Sinne hat Erwerbstätigkeit positive Auswirkungen auf den Kompetenzerwerb (Broadbridge und Swanson 2006, S. 171). So führt Erwerbstätigkeit nach Einschätzung von österreichischen Studierenden zu einem zielgerichteten Studierverhalten (44 Prozent) und zu Anregungen aus dem Beruf für das Studium (23 Prozent) (Pechar und Wroblewski 1998, S. 100). Von einer höheren Zielstrebigkeit und einer besseren Studienorganisation berichten auch Berthold und Leichsenring (o. J.b, S. 17) und Manthei und Gilmore (2005, S. 211). Interviewte Studierende aus Großbritannien geben an, ihre theoretischen Kenntnisse im Beruf einbringen zu können und die Relevanz der Lerninhalte besser zu verstehen. Aus diesem Grund müssen sie im Kurs weniger nachfragen oder sich nach Beispielen erkundigen (Evans et al. 2014, S. 86). 60 Prozent der studiennah erwerbstätigen Befragten an Wiener Fachhochschulen können ihre beruflichen Kennnisse in die Lehrveranstaltungen immer oder überwiegend 
einbringen, was eine Bereicherung sowohl für die Studierenden als auch für die Lehrenden bedeutet (Schlögl und Neubauer 2006, S. 48). Schließlich gibt es Hinweise darauf, dass erwerbstätige Studierende häufiger intrinsisch motiviert und zielstrebiger sind. Dies verstärkt sich, wenn sie studienfachnah arbeiten (Berthold und Leichsenring o. J.b, S. 15; Brandstätter und Farthofer 2003, S. 140).

Die Literatur weist überwiegend auf positive Zusammenhänge zwischen Erwerbstätigkeit und sozialer Integration in Form des Kontaktes zu Kommilitonen und des Lernens in Lerngruppen hin. Die deutsche QUEST-Studie zeigt, dass Erwerbstätige besser sozial integriert und extrovertierter sind. Dies gilt besonders für die Studierenden, die studiennah arbeiten (Berthold und Leichsenring o. J.b, S. 17). Die Beteiligung an studentischen Organisationen reduziert sich erst, wenn Studierende mehr als 30 Stunden pro Woche arbeiten (Furr und Elling 2000, S. 460). Ein Aspekt der sozialen Integration betrifft das Lernen in Gruppen. Erwerbstätigkeit hat kaum Auswirkungen auf die Häufigkeit, mit der Studierende in Gruppen lernen (jeweils etwa 21 Prozent bei Nicht- und Teilzeitberufstätigkeit). Lediglich im Falle von Vollzeitberufstätigkeit lernen nur zwölf Prozent dieser Studierenden in Gruppen (Pechar und Wroblewski 1998, S. 122). Die soziale Integration leidet somit dann, wenn Studierende im großen Umfang erwerbstätig sind (Krings et al. 2018, S. 151). In diesem Kontext sind Studien zu sehen, die von negativen Effekten wie geringerem Kontakt zu Kommilitonen und einem Gefühl der Isolation aufgrund hoher Berufstätigkeit berichten (Broadbridge und Swanson 2005, S. 242; Moreau und Leathwood 2006, S. 35).

$\mathrm{Zu}$ akademischer Integration, also der Interaktion mit Lehrenden, liegen kaum Erkenntnisse vor. Etwa 80 Prozent der erwerbstätigen Studierende an Wiener Hochschulen geben an, (eher) keine Schwierigkeiten beim Kontakt mit Lehrenden zu haben (Schlögl und Neubauer 2006, S. 51). Für deutsche Hochschulen bietet das Konstanzer Studierendensurvey Anhaltspunkte der akademischen Integration aller Studierenden als Vergleichswert. So schätzen 44 Prozent der wirtschaftswissenschaftlichen Studierenden und 56 Prozent der ingenieurswissenschaftlichen Studierenden an Fachhochschulen ihre Beziehung zu Lehrenden als (sehr) gut ein. Darüber hinaus wünschen sich 15 Prozent aller FH-Studierenden mehr intensive Betreuung durch Lehrende (Ramm et al. 2014, S. 35). Aus diesen Daten lässt sich keine schlechtere akademische Integration von erwerbstätigen im Vergleich zu nicht erwerbstätigen Studierenden ableiten.

\subsubsection{Studienerfolg}

Eine Erwerbstätigkeit in höherem Umfang reduziert die Vereinbarkeit. Doch werden diese Schwierigkeiten auch im Studienerfolg sichtbar? Nachfolgend werden die Forschungsergebnisse zur Studiendauer, Studienabbruch und Noten 
vorgestellt. Die genannten Studien fokussieren in der Regel Studierende in Volloder Teilzeitstudiengängen und damit nicht in dualen oder Weiterbildungsstudiengängen, da in letzteren Studiengängen die Vereinbarkeit von Studium und Beruf per Definition berücksichtigt werden muss.

Für Auswirkungen von Erwerbstätigkeit auf den Studienerfolg spricht, dass der zeitliche Umfang der Berufstätigkeit nicht mehr für das Studium zur Verfügung steht. Die Annahme, dass die Arbeitszeit die Zeit für das Studium im gleichen Umfang reduziert (zero-sum-Theorie), wird jedoch kritisch diskutiert. Vielmehr kann diese berufliche Belastung sowohl zulasten der Studienzeit als auch zulasten der Freizeit gehen. Möglicherweise können sich Studierende mit guten Leistungen darüber hinaus eine höhere Arbeitszeit „leisten“ (Warren 2002, S. 370). Mehrere Studien bestätigen, dass die Arbeitszeit besonders stark die Freizeit reduziert. Steigt die Arbeitszeit jedoch an, kann immer weniger Freizeit reduziert werden, und die Zeit für das Selbststudium und die Lehrveranstaltungen nimmt ab (Beerkens et al. 2011, S. 680; Oosterbeek und van den Broek 2009, S. 176). Die Sozialerhebung zeigt, dass eine Stunde Arbeitszeit die Freizeit um etwa 30 Minuten und die Studienzeit ebenfalls um 30 Minuten reduziert (Middendorff et al. 2013, S. 342). Da das Zeitinvestment in das Studium mit den akademischen Leistungen positiv zusammenhängt, kann die Reduktion der Studienzeit aufgrund von Erwerbstätigkeit negativ auf den Studienerfolg wirken (Andrietti und Velasco 2015, S. 252; Meng und Heijke 2005, S. 23). Ein weiteres Argument für die negativen Auswirkungen hoher Erwerbstätigkeit auf das Studium ist die dadurch möglicherweise leidende soziale und akademische Integration, welche für den Verbleib im Studium eine wichtige Rolle spielt (u. a. Tinto 1993). Wenn Studierende wenig(er) Zeit an der Hochschule verbringen, reduziert dies möglicherweise ihren Kontakt zu Lehrenden und Kommilitonen (Bean und Metzner 1985, S. 489; Broadbridge und Swanson 2005, S. 238). Die Berufstätigkeit kann jedoch auch positive Effekte haben, die sich auf die akademischen Leistungen auswirken. So können Studierende mit studiennahen Tätigkeiten ihre Erfahrungen und ihr Wissen aus dem Beruf im Studium anwenden und verstehen besser, wozu sie die Studieninhalte im Berufsleben benötigen (Beerkens et al. 2011, S. 686; Donaldson und Graham 1999, S. 28).

Zahlreiche Studien beschäftigen sich mit den Auswirkungen von Erwerbstätigkeit auf unterschiedliche Facetten des Studienerfolgs. Dabei ist die Literatur aus Großbritannien und den USA besonders umfangreich; mehrere Studien liegen auch aus europäischen Ländern und aus dem deutschsprachigen Raum vor. Nachfolgend werden - in der Regel multivariate - Ergebnisse zur Berufstätigkeit von Hochschulstudierenden vorgestellt. Die umfangreiche Literatur zu Auswirkungen von Berufstätigkeit in US-amerikanischen 
Highschools bleibt aufgrund der nicht ausreichenden Vergleichbarkeit zwischen Schul- und Hochschulbildung unberücksichtigt.

Nationale und internationale Studien sind sich zunächst einig darüber, dass umfangreiche Berufstätigkeit die Studiendauer verlängert. $\mathrm{Ab}$ welcher Stundenanzahl der studienzeitverlängernde Effekt einsetzt, ist jedoch je nach Untersuchung und Landeskontext unterschiedlich. Body et al. (2014, S. 9) zeigen am Beispiel von Frankreich, dass ab acht Stunden Erwerbstätigkeit ${ }^{4}$ ein negativer Effekt auf das erfolgreiche Absolvieren der ersten beiden Studiensemester einsetzt, der sich ab 16 Stunden noch verstärkt. Furr und Elling (2000, S. 467) befragten Studierende aus den USA nach ihrem Studienfortschritt und zeigen bivariat, dass bei zehn bis 29 Stunden Arbeitszeit ein geringer, bei 30 bis 39 Stunden ein mittlerer und ab 39 Stunden ein hoher negativer Effekt auftritt. Beerkens et al. (2011, S. 687) und Triventi (2014, S. 10) weisen auf einen stark negativen Effekt ab 25 Stunden Arbeitszeit (Estland) bzw. 20 Stunden (Italien) hin. Im deutschsprachigen Raum hat Jirijahn (2007, S. 303) die Studiendauer im wirtschaftswissenschaftlichen Studium anhand der Studiendauer bis zum Vordiplom analysiert. Der Autor gibt jedoch keine Stundenzahl an, sondern die subjektiven Belastungen durch Erwerbstätigkeit und findet negative Effekte dieser Belastung auf die Studiendauer. Nach bivariaten Analysen von Brandstätter und Farthofer (2003, S. 140) in Österreich reduziert sich die Anzahl der abgelegten Prüfungen pro Semester ab sechs Stunden Erwerbstätigkeit leicht und ab 18 Stunden stark. Die genannten Studien stimmen darin überein, dass ein nichtlinearer Effekt existiert, der negative Auswirkungen von Erwerbstätigkeit erst $\mathrm{ab}$ einer höheren Stundenanzahl eindeutig ausweist. Als Gründe hierfür werden die reduzierte Zeit für Lehrveranstaltungen und Selbstlernzeit genannt. Weiterhin gibt es Hinweise darauf, dass die Branche, in der Studierende erwerbstätig sind, sowie die Flexibilität neben der Stundenzahl einen wichtigen Faktor ausmachen: Arbeiten Studierende studiennah oder im öffentlichen Sektor, so verlängert dies die Studienzeit kaum (Body et al. 2014, S. 9). Darüber hinaus schätzen Studierende die Vereinbarkeit von wissenschaftlichen Tätigkeiten mit dem Studium deutlich besser ein als beispielsweise von Aushilfstätigkeiten (Mertens 2013, S. 47).

$\mathrm{Ob}$ berufstätige Studierende ihr Studium häufiger abbrechen, ist nicht eindeutig geklärt. Viele Studien weisen der Berufstätigkeit bzw. schlechter Vereinbarkeit von Beruf und Studium allerdings eine Relevanz hinsichtlich des Studienabbruchs $\mathrm{zu}$, auch wenn Berufstätigkeit in der Regel nur einen von mehreren Abbruchgründen darstellt (Georg 2008, S. 200; Pohlenz und Tinsner 2004, S. 110). Eine Kombination aus finanziellen Engpässen, fehlender

4 Die nachfolgenden Stundenangaben beziehen sich auf die wöchentliche Arbeitszeit. 
Vereinbarkeit von Studium und Beruf sowie einer zu langen Studiendauer ist für 19 Prozent der Studienabbrecherinnen und Studienabbrecher ausschlaggebend für den Abbruch (Heublein et al. 2010, S. 19). Es fehlen jedoch Analysen dazu, wie sich der Umfang der Berufstätigkeit auf den Abbruch auswirkt. Eine schlecht mit dem Studium zu vereinbarende Berufstätigkeit hängt offenbar in Deutschland mit dem Studienabbruch zusammen. Internationale Studien wie diejenige von Beerkens et al. (2011, S. 687) finden hingegen keine signifikanten Auswirkungen von Berufstätigkeit darauf, ob Studierende ihr Studium zu Ende führen oder abbrechen.

$\mathrm{Zu}$ dem Einfluss von Berufstätigkeit auf Noten liegen divergierende Ergebnisse vor. Der eine Bereich der Forschung weist negative Effekte aus. Callender (2008, S. 370) findet lineare negative Effekte der Berufstätigkeit auf den Studienerfolg in sechs englischen Universitäten. Brandstätter und Farthofer (2003, S. 140) zeigen für Österreich, Krings et al. (2018, S. 151) für Deutschland und Miller et al. (2008, S. 678) für die USA, dass signifikante negative Effekte erst ab 18 bzw. 20 Stunden Berufstätigkeit auftreten. Diese Autoren gehen davon aus, dass unter anderem die fehlende Zeit für das Studium die akademische Leistung schmälert (siehe auch Evans et al. 2014, S. 87). Ergebnisse einer USamerikanischen Studie unter Studierenden der sozialen Arbeit von Hawkins et al. (2005, S. 17) legen nahe, dass die wahrgenommene Belastung bzw. eine schlechte Vereinbarkeit die negativen Effekte von Berufstätigkeit auf Noten besser abbildet als die konkrete Stundenzahl.

Ein weiterer Forschungsbereich zeigt, dass eine geringe Erwerbstätigkeit zu einer Verbesserung der Studienleistungen führen kann. Ein möglicher Grund ist, dass die zeitliche Belastung durch die Erwerbstätigkeit niedrig ist und die Erfahrungen im Studium genutzt werden können (Meng und Heijke 2005, S. 23). Darolia (2014, S. 44) zeigt mit Daten aus den USA bivariat einen positiven Effekt von Erwerbstätigkeit im Umfang von bis zu acht Stunden. Anhand des Nationalen Bildungspanels in Deutschland verdeutlicht Sprietsma (2015, S. 12), dass Erwerbstätigkeit unter 15 Stunden einen positiven Effekt auf Noten hat.

Schließlich nennt ein Anteil der Studierenden die besseren Berufschancen als Grund für die Erwerbstätigkeit. Häkkinen (2006, S. 179) zeigt in einer finnischen Längsschnittstudie, dass studentische Erwerbstätigkeit tatsächlich mit einer geringeren Arbeitslosigkeit und einem höheren Gehalt zusammenhängt. Erwerbstätigkeit führt somit zwar zu längeren Studienzeiten, kann aber auch positive Auswirkungen auf Noten und den Berufseinstieg haben. 


\subsubsection{Anforderungen an das Studium}

Anhand der Aspekte struktureller Studierbarkeit (siehe Kapitel 2.1) werden nachfolgend die Anforderungen erwerbstätiger Studierender an ein Studium beschrieben. Strukturelle Studierbarkeit kann dabei anhand der folgenden Kategorien analysiert werden:

- Ort und Zeit von Veranstaltungen,

- die Verteilung des Arbeitsaufwandes,

- die Möglichkeit, das Studium zu unterbrechen und die Dauer anzupassen, sowie

- die Flexibilität des Formats (z. B. Teilzeit, Fernstudium).

Die Literatur zu Anforderungen von berufstätigen Studierenden ist in Tabelle 4 komprimiert dargestellt und wird im Folgenden kurz zusammengefasst. Besonders wichtig ist für erwerbstätige Studierende eine Mischung aus Flexibilität und Planbarkeit. Die Flexibilität kann sich in der orts- und zeitunabhängigkeit von Veranstaltungen durch E-Learning, geringe Anwesenheitspflichten und hohe Wahlmöglichkeiten ausdrücken. Auch die Zeitpunkte und Fristen für Prüfungen sowie die Unterbrechung und Verlängerung des Studiums flexibilisieren das Studium. Planbarkeit bezieht sich auf die Sicherheit, in vorher bekannten Zeitfenstern einer Berufstätigkeit nachgehen zu können. Auch der Wechsel in ein Teilzeitstudium bietet die Sicherheit, ohne Fristverletzungen länger studierenden zu können.

Sind Studierende in größerem Umfang berufstätig, so sind sie auf angemessene Öffnungszeiten von Serviceeinrichtungen angewiesen. Dies betrifft sowohl den Studierendenservice in Form von Prüfungsämtern als auch Bibliotheken und Sprechstunden. Online- oder Telefonberatungen sowie die verstärkte Nutzung von internetbasierten Serviceportalen (z. B. HIS-in-One, Datenlotsen) ermöglichen zusätzliche Ortsunabhängigkeit. 
Tabelle 4 Anforderungen von berufstätigen Studierenden an Studienstrukturen.

\begin{tabular}{|c|c|c|}
\hline Thema & Anforderung & \multirow{19}{*}{$\begin{array}{l}\text { Quellen } \\
\text { Hall (2010), } \\
\text { Pechar und } \\
\text { Wroblewski } \\
\text { (1998), } \\
\text { Moreau und } \\
\text { Leathwood } \\
\text { (2006), } \\
\text { Nienhüser et al. } \\
\text { (2000), } \\
\text { Schlögl und } \\
\text { Neubauer (2006) } \\
\text { Busch- } \\
\text { Karrenberg } \\
\text { (2014), } \\
\text { Buß et al. (2015) }\end{array}$} \\
\hline Ort und Zeit von & E-Learning, geringe & \\
\hline \multirow{5}{*}{$\begin{array}{l}\text { Lehrveranstaltun } \\
\text { gen }\end{array}$} & Anwesenheitspflicht, Unterlagen & \\
\hline & zum Selbststudium, & \\
\hline & Blockveranstaltungen, langfristig & \\
\hline & und sich nicht ändernde & \\
\hline & $\begin{array}{l}\text { Veranstaltungszeiten, freie Zeiten } \\
\text { oder Tage für Berufstätigkeit }\end{array}$ & \\
\hline Umfang der & Flexible Prüfungstermine und & \\
\hline Präsenzlehre und & Fristen, viele Wahlmodule, & \\
\hline Verteilung des & Anrechnung von beruflichen & \\
\hline Arbeitsaufwands & Kompetenzen auf das Studium & \\
\hline $\begin{array}{l}\text { Studienunterbrec } \\
\text { hung und }\end{array}$ & $\begin{array}{l}\text { Sandwich-Studium mit Auszeiten } \\
\text { für Berufstätigkeit, Urlaubssemester }\end{array}$ & \\
\hline Studiendauer & für Berufstätigkeit & \\
\hline Flexibilität des & Teilzeitstudium, Fernstudien(- & \\
\hline Studienformats & Anteile) & \\
\hline Beratung und & Flexible/lange Öffnungszeiten der & \\
\hline Betreuung & Serviceeinrichtungen (z. B. & \\
\hline & Prüfungsamt, Bibliothek), Beratung & \\
\hline & $\begin{array}{l}\text { zur Vereinbarkeit, virtuelle } \\
\text { Beratung und Service }\end{array}$ & \\
\hline
\end{tabular}

\subsection{Studierende mit Kind}

\subsubsection{Lebens- und Studiensituation}

An deutschen Hochschulen haben 6 Prozent aller Studierenden Kinder. Die durchschnittliche Kinderanzahl beträgt dabei 1,6. Die Kinder sind überwiegend im Kindergartenalter. 46 Prozent dieser Studierendengruppe haben Kinder im Alter von bis zu drei Jahren, weitere 16 Prozent haben Kinder im Alter von vier bis sechs Jahren.

Studierende mit Kind sind häufig in Teilzeit- oder berufsbegleitenden Studiengängen eingeschrieben (jeweils zehn Prozent). Studierende mit Kind weisen mit 35 Jahren ein höheres Durchschnittsalter auf als die Studierenden ohne 
Kind mit durchschnittlich 24 Jahren (Middendorff et al. 2017, S. 27 f.). Darüber hinaus haben 40 Prozent der Studierenden ihr jüngstes Kind vor Studienbeginn bekommen und konnten somit bei der Wahl des Studiengangs mögliche Anforderungen an die Vereinbarkeit mit der Familie schon berücksichtigen (Middendorff et al. 2013, S. 480).

Tabelle 5 Studierende mit Kind in Deutschland. Eigene Darstellung mit Daten aus Middendorff et al. (2013, 2017).

\begin{tabular}{lll}
\hline Studierende mit Kind & & \\
\hline Quote & Gesamt & $6 \%$ \\
\hline Studiengangart & Bachelor & $5 \%$ \\
& Master & $6 \%$ \\
\hline Berufstätigkeit* & Ohne Kind & $62 \%$ \\
& Mit Kind & $59 \%$ \\
\hline Durchschnittliches & Ohne Kind & 24 Jahre \\
Alter & Mit Kind & $35 \mathrm{Jahre}$ \\
\hline Geschlecht & Frauen & $6 \%$ \\
& Männer & $5 \%$ \\
\hline Bildungshintergrund* & Niedrig, ohne Kind & $9 \%$ \\
& Niedrig, mit Kind & $16 \%$ \\
\hline
\end{tabular}

* Zeitpunkt 2012. Alle Andere Daten: 2016

Aufgrund der langen Schul- und Studienzeit, der finanziellen Abhängigkeit von der Familie und Schwierigkeiten in der Vereinbarkeit von Studium und Sorgeaufgaben bekommen Studierende das erste Kind deutlich später als junge Frauen mit einer Berufsausbildung. 48 Prozent der Frauen mit Sekundarabschluss, aber nur 24 Prozent der Frauen mit Hochschulabschluss gebären vor dem 28. Lebensjahr ihr erstes Kind (Ochel 2006, S. 11). Für die Politik ist die Vereinbarkeit von Studium und Familie daher ein wichtiges Ziel, um die geringen Geburtenraten von Akademikerinnen zu erhöhen (BMBF 2013, Vorwort).

Studierende mit und ohne Kind sind mit jeweils etwa 60 Prozent ähnlich häufig erwerbstätig. Es gibt jedoch Unterschiede zwischen den Geschlechtern: Etwa die Hälfte der studierenden Mütter arbeitet - bei ihren Kommilitoninnen ohne Kind sind es zwei Drittel. Die in dieser Arbeit betrachteten Studiengänge sind überwiegend der Betriebswirtschaft, der Informatik und dem Sozial- und Gesundheitswesen zuzuordnen. Diesen Fächer unterscheiden sich deutlich hinsichtlich des Anteils der Studierenden mit Kind: In der Medizin und im Gesundheitsbereich gibt es mit 6 Prozent etwa doppelt so viele Eltern wie in den Rechts- und Wirtschaftswissenschaften. Schließlich unterscheiden sich die 
Bildungsbiografien von studierenden Eltern und Studierenden ohne Kind. Der Bildungshintergrund von Studierenden mit Kind ist häufiger als „niedrig“ einzustufen, und sie waren vor dem Studium öfter berufstätig (Dippelhofer-Stiem 2012, S. 418; Middendorff et al. 2013, S. 491 ff.).

\subsubsection{Studienerfolg}

Bisher liegen keine Daten zu Studienabbruch und Studiengangwechsel von Studierenden mit Pflegeaufgaben vor. Die Sozialerhebung weist Pflege allerdings als einen Grund für Studienunterbrechungen aus, der von 5 Prozent der Studierenden angegeben wird. Damit stellt Pflege zwar auf den ersten Blick einen seltenen Unterbrechungsgrund dar, sie ist aber angesichts des geringen Anteils an pflegenden Studierenden ein sehr häufiger Grund. In Familien mit niedrigem Bildungshintergrund ist es besonders üblich, Familienangehörige selbst $\mathrm{zu}$ pflegen. Studierende mit niedrigem Bildungshintergrund (9 Prozent) unterbrechen ihr Studium zur Pflege daher doppelt so oft wie Studierende mit gehobenem oder hohem Bildungshintergrund (Klaus und Tesch-Römer 2014, 2 ff.; Middendorff et al. 2017, S. 82). Auch wenn eine Studienunterbrechung bei hoher Belastung eine sinnvolle Maßnahme sein kann, weisen Erkenntnisse aus Forschung zur Vereinbarkeit von Beruf und Pflege auf die Ausgleichsfunktion der Berufstätigkeit bzw. des Studiums zur Pflegeverantwortung hin (Franke und Reichert o. J., S. 49; Reichert 2012, S. 326). Der soziale Kontakt zu Kommilitoninnen und Kommilitonen und das Lernen im Studium können für Studierende mit Pflegeaufgaben somit einen wichtigen emotionalen und fachlichen Ausgleich zur Pflege bieten. Die Erhöhung der Vereinbarkeit von Pflege und Studium ist daher als für die betroffenen Personen wichtig einzuschätzen.

\subsubsection{Studierverhalten}

Das Studierverhalten der Studierenden mit Kind ist durch die Herausforderung geprägt, die Kinderbetreuung mit Präsenzveranstaltungen und Selbstlernzeit in Einklang zu bringen. Studierende Mütter haben insbesondere Probleme mit Veranstaltungszeiten (66 Prozent), Flexibilität (54 Prozent) und der Unterstützung durch die Lehrenden (42 Prozent) (Helfferich et al. 2007, S. 127). Probleme mit der Anwesenheit bei Lehrveranstaltungen oder Freiräumen für Selbstlernzeit erhöhen sich, wenn Studierenden nur unzureichende Betreuungsmöglichkeiten zur Verfügung stehen oder wenn sie alleinerziehend sind und daher die Unterstützung durch den Partner wegfällt (Middendorff et al. 2013, S. 487). Da nur 17 Prozent der Studierenden mit Kind in Baden-Württemberg angeben, mit der 
Kinderbetreuung zufrieden zu sein, ist die Vereinbarkeit ein ernst zu nehmendes Problem (Helfferich et al. 2007, S. 26). Dies bestätigen auch Daten aus Großbritannien, da hier die Hälfte der Studierenden mit Kind Lehrveranstaltungen aufgrund fehlender Kinderbetreuung verpasst (National Union of students UK 2009, S. 28).

Die Vereinbarkeit bezieht sich jedoch nicht nur auf Studium und Familie, sondern häufig auch auf die Berufstätigkeit. Durch die unterschiedlichen Anforderungen der Verpflichtungen kann eine Zerrissenheit entstehen, die Greenhaus und Benthell (1985, S. 77) als Interrollenkonflikt beschreiben: ,,a form of interrole conflict in which the role pressures from the work and family domains are mutually incompatible in some respect". Die Vereinbarkeit kann daher als work-life-learn balance beschrieben werden, bei der Konflikte aufgrund von zeitlichen Restriktionen entstehen (Lowe und Gayle 2007, S. 228). Zeit wird dabei als knappes Gut wahrgenommen, von dem generell zu wenig vorhanden ist (Helfferich et al. 2007, S. 96). Für viele Studierende ist es eine besonders Herausforderung, dass sowohl das Studium als auch Kinder jederzeit Anforderungen an sie stellen. Die Anforderungen von Kindern konkurrieren mit Selbstlernzeit und Prüfungsvorbereitung, für die die Studierenden längere ungestörte Zeiträume benötigen (Dippelhofer-Stiem 2012, S. 420). Welche der Anforderungen prioritär bedient werden, hängt auch mit den Rollenverständnissen der Studierenden und des Umfelds zusammen (Middendorff 2003, S. 18; Schiller 2013, S. 101; Sieverding 1992, S. 156). Wegen der konkurrierenden Verpflichtungen können nur ein Drittel der Studierenden ihre Freizeitinteressen verwirklichen (Helfferich et al. 2007, S. 98). Eine US-amerikanische Studie zur Vereinbarkeit von Arbeit, Studium und Familie zeigt, dass die Konflikte zwischen Studium und Arbeit sowie Studium und Beruf unterschiedlich stark wahrgenommen werden: Der Konflikt zwischen Familie und Studium ist größer als der zwischen Arbeit und Studium (Hammer et al. 1998, S. 224). Ein Grund hierfür sind die ständigen Bedürfnisse von Kindern, wohingegen Berufstätigkeit in der Regel auf feste Arbeitszeiten begrenzt bleibt. Auch wenn Studierende die Vereinbarkeit als großes Problem ansehen, ist das Studium trotzdem für viele Studierende mit Kind realisierbar. Sollen Studierende mit Kind einschätzen, ob Studium und Familie miteinander vereinbar sind, so geben sie einen Mittelwert von 2,6 auf einer 5er-Skala an $(5=$ schlechte Vereinbarkeit). Somit schätzen Studierende mit Kind die Machbarkeit eines Studiums mit gleichzeitiger familiärer Belastung positiver ein als Studierende ohne Kind $(\mathrm{MW}=3,1)$ (Middendorff 2003, S. 22). Bei dieser Interpretation der Mittelwerte ist jedoch zu beachten, dass nur noch diejenigen Studierenden mit Kind an der Hochschule verbleiben, die Studium und Familie vereinen können. 
Ein gutes Verständnis der Lehrenden für die Lebenssituation der Eltern und guter Kontakt zu Kommilitonen können das Studium erleichtern. Zu dieser sozialen und akademischen Integration in das Studium liegen Befunde vor, die zunächst eine schlechtere Integration der Studierenden mit Kind vermuten lassen. Die Hälfte der an baden-württembergischen Hochschulen befragten Eltern fühlt sich der Hochschule „nicht mehr richtig zugehörig“ (Helfferich et al. 2007, S. 45). Weitere Forschungsergebnisse zeichnen jedoch ein differenziertes Bild. So reichen beispielsweise die Reaktionen der Lehrenden auf Schwangerschaft und Elternschaft von Studierenden von hohem Entgegenkommen bis hin zu Abwehr. Lehrende unterstützen Studierende mit Kind beispielsweise durch ihre Offenheit und Bereitschaft, Fristen zu verlängern und Abwesenheit auszugleichen. Einige Lehrende sehen das Studium jedoch auch als oberste Priorität, der Familie oder Beruf unterzuordnen seien (Gräwe-Meier und Müller 2008, S. 81; Weber et al. 2008, S. 218). Bundes- oder landesweite quantitative Studien zeigen, dass ein Viertel (Middendorff 2003, S. 23) bis ein Drittel (Helfferich et al. 2007, S. 23) der Dozentinnen und Dozenten ein (eher) hohes Verständnis für die Situation von Studierenden mit Kind zeigen. Aus Großbritannien liegen mit 39 Prozent ähnliche Werte vor (National Union of students UK 2009, S. 32). Diesen eher schlechten Bewertungen von Studierenden mit Kindern stehen Ergebnisse des Konstanzer Studierendensurveys gegenüber, die tendenziell abmildernde Auswirkungen der Elternschaft auf die Schwierigkeiten im Kontakt mit Lehrenden zeigen. Auch haben Studierende mit Kind etwas häufiger Kontakt zu Professoren als Studierende ohne Kind (Dippelhofer-Stiem 2012, S. 422 ff.).

Bezogen auf die soziale Integration berichten Studierende mit Kind, dass sich der Kontakt zu anderen Kommilitoninnen und Kommilitonen mit Kind verstärkt und einige der Freundschaften mit Kommilitonen ohne Kinder enden (Berthold und Leichsenring o. J.a, S. 14; Weber et al. 2008, S. 212). Studierende mit Kind haben signifikant seltener Kontakt zu Kommilitoninnen und Kommilitonen im eigenen Fach oder in anderen Fächern (Dippelhofer-Stiem 2012, S. 425). Dieser geringere Kontakt ist umso kritischer, da viele Studierende mit Kind ihren Kontakt zu Mitstudierenden und Lehrenden hoch priorisieren (Schiller 2013, S. 135). Eine geringere Anwesenheit an der Hochschule und Probleme, an Gruppenarbeiten teilzunehmen, bedingen teilweise eine schlechtere soziale Integration der Studierenden mit Kind in die Hochschule. Studierende mit Kind sind daher leicht schlechter sozial integriert als Studierende ohne Kind (Berthold und Leichsenring o. J.a, S. 16; Buß, Erbsland et al. 2018, S. 122 f.).

Neben den Herausforderungen weisen mehrere Studien darauf hin, dass Studierende auch für das Studium nützliche Fähigkeiten aus ihrer Elternschaft ziehen können. Denn durch die komplexe Organisation des Familien- und Studienalltags in Kombination mit geringen Zeitressourcen lernen Studierende mit 
Kind möglicherweise stringenter und verfügen über ein besseres Zeitmanagement (Gräwe-Meier und Müller 2008, S. 29; Krenmayr 2008, S. 23; Müller et al. 2015, S. 18). Krenmayr (2008) zeigt die Unterschiede in der Persönlichkeit der auf den ersten Blick homogenen Gruppe der Studierenden mit Kind auf. Anhand der subjektiv positiven oder negativen Konnotation der Elternschaft und der Erholungsmöglichkeiten bzw. Belastungen der Studierenden identifiziert die Autorin Persönlichkeitsfaktoren wie Gewissenhaftigkeit, emotionale Stabilität oder Extraversion als Faktoren, die den Studienverlauf positiv beeinflussen (Krenmayr 2008, S. 53). Ob Studierende mit Kind andere Persönlichkeitsmerkmale, etwa eine höhere Gewissenhaftigkeit, aufweisen, kann aufgrund der fehlenden Vergleichsgruppe von Studierenden ohne Kind nicht ermittelt werden. Kurz- und langfristige Zeitplanung, Gewissenhaftigkeit und die Disziplin, Zeit sinnvoll zu nutzen und auch unangenehme Tätigkeiten zu Ende zu bringen, haben einen positiven Einfluss auf akademische Leistungen (Britton und Tesser 1991, S. 408). Sie sind gleichzeitig Elemente der Selbstlernkompetenz (Wild und Wild 2012, S. 16). Möglicherweise ist die Erfahrung mit Zeitmanagement einer der Gründe für die guten akademischen Leistungen von vielen Studierenden mit Kind (Helfferich et al. 2007, S. 166; Schiller 2013, S. 128; Scott et al. 1998, S. 222). Quantitativ empirisch belegte Aussagen zu dieser Hypothese fehlen jedoch bis dato. Einige Studierende berichteten in Interviews von Konzentrationsschwierigkeiten aufgrund zeitlicher Belastungen, andere Studierende hingegen von positiven Auswirkungen ihres Kindes auf ihre Lernleistung:

„Das ist auch, man nutzt die Zeit, die man dann zum Lernen hat, wirklich auch effektiv. Das ist ein großer Unterschied zu vielen anderen, also ich habe selten das Problem, dass ich mich nicht dransetzen kann an meine Sachen." (Weber et al. 2008, S. 195).

Die Studienmotivation der Studierenden mit Kind stellt einen weiteren wichtigen Studienerfolgsfaktor dar (Heublein et al. 2010, S. 28). Studierende mit Kind geben besonders oft an, ein Rollenvorbild für ihre Kinder sein zu wollen und gerne zu lernen (Osborne et al. 2004, S. 297). Darüber hinaus sind sie stärker intrinsisch motiviert, möchten sich beruflich beweisen, fachlichen Problemen nachgehen und sich weiterentwickeln. Karriere und Geld spielen eine geringere Rolle als bei Studierenden ohne Kind (Berthold und Leichsenring o. J.a, S. 16; Hess et al. 2014, S. 8; Middendorff 2003, S. 13; Scott et al. 1998, S. 222). 


\subsubsection{Studienerfolg}

Der Studienerfolg der Studierenden mit und ohne Kind unterscheidet sich insbesondere in der Studiendauer und der Häufigkeit eines Studiengangwechsels. So geben 87 Prozent der Studierenden mit Kind an Hochschulen in BadenWürttemberg an, dass sich ihr Studium durch die Elternschaft verlängert habe. An Fachhochschulen beträgt die Verlängerung durchschnittlich zwei Semester (Helfferich et al. 2007, S. 165). In Wiener Hochschulen ist eine Verlängerung um im Median vier Semester zu beobachten (Krenmayr 2008, S. 62). Verlängerungen gehen dabei insbesondere auf Studienunterbrechungen durch Schwangerschaft und Geburt sowie auf die Kinderbetreuung zurück. Zweifel an der Sinnhaftigkeit des Studiums oder der Wunsch, andere Erfahrungen $\mathrm{zu}$ machen, führen bei Studierenden mit Kind hingegen seltener zu Unterbrechungen als bei Studierenden ohne Kind. Verlängerungen zwischen Studienstart und Studienabschluss entstehen unter anderem durch Urlaubssemester für Geburt und Elternzeit, die jedoch nicht alle Mütter in Anspruch nehmen. Ein Grund hierfür ist das Ziel, das Studium zügig abzuschließen (Weber et al. 2008, S. 194). Verlassen Studierende einen Studiengang, ist dies insbesondere auf Studiengang- und Hochschulwechsel zurückzuführen. Studierende mit und ohne Kind unterscheiden sich nicht darin, wie häufig sie die Hochschule wechseln. Nur der Studiengangwechsel kommt bei Studierenden mit Kind etwas häufiger vor als bei Studierenden ohne Kind (Middendorff et al. 2013, 499 f.). Ob Studierende mit Kind häufiger aus dem Hochschulsystem ausscheiden, ist nicht eindeutig erfasst. Einerseits geben drei Prozent der Abbrechenden an, dass sie ihr Studium wegen der Unvereinbarkeit von Studium, Schwangerschaft und Kinderbetreuung nicht weiterführen konnten (Heublein et al. 2010, S. 41). Andererseits zeigen zwei deutsche Studien, dass die Elternschaft nicht mit einem höheren Studienabbruch zusammenhängt (Buß, Erbsland et al. 2018, S. 123; Stoessel et al. 2015, S. 12). Die Herausforderungen für Studierende mit Kind liegen insbesondere in der Vereinbarkeit von Studium und Familie; ihre Leistungsfähigkeit schätzen die Studierenden mit Kind hoch ein. So geben $35 \%$ an, ihre Noten lägen über dem Durchschnitt des Faches (Helfferich et al. 2007, S. 166).

\subsubsection{Anforderungen an das Studium}

Mehrere Studien haben sich mit der Frage beschäftigt, welche Probleme Studierende mit Kind im Studienalltag haben und welche Maßnahmen und Strukturen sie nach Einschätzung der Befragten unterstützen würden. Für einen systematischen Überblick über die Aktivitäten von Hochschulen im Bereich der Familienfreundlichkeit sei auf eine von der GESIS durchgeführte bundesweite 
Studie verwiesen (Kunadt et al. 2014). In der Tabelle 6 sind anhand der Kriterien struktureller Studierbarkeit die Anforderungen der Studierenden mit Kind an Studienstrukturen überblicksartig dargestellt. Studierende mit Kind haben widersprüchliche Anforderungen an Studienstrukturen. Auf der einen Seite ist für sie eine langfristige Planbarkeit wichtig, die sich unter anderem in der frühen Bekanntgabe von Lehrveranstaltungszeiten und Prüfungen oder der geplanten Streckung des Studiums in Teilzeitform bemerkbar macht. Durch Schwangerschaft, Krankheit des Kindes oder Engpässe bei der Betreuung benötigen sie andererseits eine hohe Flexibilität, um Abwesenheiten auszugleichen. Viele Wahlmöglichkeiten, mehrere Möglichkeiten zur Erbringung von Leistungen, und flexible Beurlaubungen helfen Eltern, kurz- und mittelfristig den Studien- und Familienalltag in Einklang zu bringen (Müller et al. 2015, S. 21; Schiller 2013, S. 129). Kinderbetreuung, die auch flexibel und zu Randzeiten genutzt werden kann, ist eine der wichtigsten Forderungen von studierenden Eltern. Die Kinderbetreuung ist der einzige Aspekt struktureller Studierbarkeit, für den die Hochschule nicht hauptverantwortlich ist. Für die Einrichtung von Kindertagesstätten sind die Studierendenwerke oder städtische Einrichtungen zuständig. Einige Projekte von Hochschulen versuchen allerdings, fehlende Kindertagesstätten durch die Finanzierung von Tagesmüttern und Tagesvätern oder von Studierenden und Vereinen organisierte Betreuung auszugleichen (Hochschule Magdeburg 2018; Uhlig und Wilke 2008, S. 3). 
Tabelle 6 Zusammenfassung der Anforderungen von Studierenden mit Kind an die Studienstrukturen.

\begin{tabular}{|c|c|c|}
\hline Thema & Anforderung & Quellen \\
\hline $\begin{array}{l}\text { Ort und Zeit von } \\
\text { Lehrveranstal- } \\
\text { tungen }\end{array}$ & $\begin{array}{l}\text { E-Learning, keine } \\
\text { Überschneidungen, viele } \\
\text { Wahlmöglichkeiten, Lage der } \\
\text { Veranstaltungen innerhalb der } \\
\text { Betreuungszeiten, bevorzugte } \\
\text { Seminarplatzvergabe, frühzeitige } \\
\text { Bekanntgabe von Veranstaltungs- } \\
\text { und Prüfungszeiten, wenig Pausen } \\
\text { zwischen Veranstaltungen, E- } \\
\text { Learning }\end{array}$ & $\begin{array}{l}\text { Kunadt et al. } \\
\text { (2014), } \\
\text { Schiller (2013), } \\
\text { Gräwe-Meier und } \\
\text { Müller (2008), } \\
\text { Helfferich et al. } \\
\text { (2007), } \\
\text { National Union of } \\
\text { students UK } \\
\text { (2009), }\end{array}$ \\
\hline $\begin{array}{l}\text { Umfang der } \\
\text { Präsenzlehre und } \\
\text { Verteilung des } \\
\text { Arbeitsaufwands }\end{array}$ & $\begin{array}{l}\text { Blockveranstaltungen, Prüfungen } \\
\text { und Abgabefristen nicht in oder } \\
\text { direkt nach Ferien oder Feiertagen }\end{array}$ & $\begin{array}{l}\text { Lidl (2013), } \\
\text { Dippelhofer- } \\
\text { Stiem (2009), } \\
\text { BMFSFJ (2012), }\end{array}$ \\
\hline $\begin{array}{l}\text { Studienunterbrech } \\
\text { ung und } \\
\text { Studiendauer }\end{array}$ & $\begin{array}{l}\text { Beurlaubung und ggf. Schreiben } \\
\text { von Prüfungen in der Beurlaubung, } \\
\text { Verlängerung der Regelstudienzeit, } \\
\text { Ausnahmen wegen Krankheit des } \\
\text { Kindes }\end{array}$ & $\begin{array}{l}\text { Buß, Erbsland et } \\
\text { al. (2018), } \\
\text { Weber et al. } \\
\text { (2008), } \\
\text { Uhlig und Wilke }\end{array}$ \\
\hline $\begin{array}{l}\text { Flexibilität des } \\
\text { Studienformats }\end{array}$ & Teilzeitstudium & \\
\hline $\begin{array}{l}\text { Beratung und } \\
\text { Betreuung }\end{array}$ & $\begin{array}{l}\text { Kinderbetreuung, Informations- } \\
\text { und Beratungsangebote, Beratung } \\
\text { zu Studienfinanzierung }\end{array}$ & \\
\hline
\end{tabular}

\subsection{Studierende mit Pflegeaufgaben}

\subsubsection{Lebens- und Studiensituation}

Studierende mit Pflegeaufgaben sind ebenso wie Studierende mit Kind eine relativ kleine Gruppe, deren Anteil sich jedoch mit steigendem Lebensalter der Studierenden erhöht. Die Pflegenden bilden eine bisher in der Hochschulforschung wenig beachtete Gruppe. So erhebt die Sozialerhebung als wichtige Quelle für bundesweite Daten $\mathrm{zu}$ Studierenden bisher keine Informationen zu Studierenden mit Pflegeaufgaben (Kunadt et al. 2014, 44, 47). Studien legen Pflege unterschiedlich eng oder weit aus und differenzieren unter 
anderem zwischen körperlicher Pflege und (emotionalen) Betreuungsleistungen (Überblick siehe Franke und Reichert o. J., S. 12). Insgesamt pflegen vier Prozent aller Deutschen einen oder mehrere Angehörige. Wenn Betreuung und Pflege zusammengezählt werden, geben mit 7,9 Prozent deutlich mehr Menschen in der Altersklasse von 14 bis 29 Jahren an, sich entsprechend um Angehörige zu kümmern (Vogel 2016, S. 2). Weil ältere Menschen häufiger Angehörige pflegen, ist bei Studierenden von einem unterdurchschnittlichen Anteil an Pflegenden auszugehen (Klaus und Tesch-Römer 2014, S. 2). Die QUEST-Erhebung des CHE bestätigt dies und zeigt, dass lediglich 3,1 Prozent der Studierenden Angehörige pflegen (Berthold und Leichsenring o. J.a, S. 20).

Sowohl unter den Studierenden als auch im bundesweiten Schnitt pflegen diejenigen Menschen häufiger Angehörige, die nach Deutschland zugewandert sind und deren Eltern keinen Hochschulabschluss aufweisen. Ein hohes Bildungsniveau hängt mit einer geringeren Pflegerate und weniger Wochenstunden der Pflege zusammen (Berthold und Leichsenring o. J.a, 20 f.; Klaus und Tesch-Römer 2014, S. 5). Studierende mit Pflegeaufgaben sind mit 25,8 Jahren etwa zwei Jahre älter als Studierende ohne Pflegeaufgaben. Sie sind oft mehrfach belastet, da sie ebenfalls häufiger Kinder haben und einer Berufstätigkeit nachgehen. Fast 60 Prozent der Pflegenden in Deutschland sind weiblich (Keck und Saraceno 2009, S. 8).

Tabelle 7 Studierende mit Pflegeaufgaben in Deutschland. Eigene Darstellung mit Daten aus: Keck und Saraceno (2009), Berthold und Leichsenring (o. J.a), Klaus und TeschRömer (2014).

\begin{tabular}{lll}
\hline \multicolumn{3}{l}{ Studierende mit Pflegeaufgaben von Angehörigen } \\
\hline Quote & Gesamt & $3,1 \%$ \\
\hline Durchschnittliches & Ohne & 23,6 Jahre \\
Alter & Pflegeverantwortung & Mit Pflegeverantwortung \\
& 25,8 Jahre \\
\hline Geschlecht & Mrauen & $60 \%$ der Pflegenden \\
& Männer & $40 \%$ der Pflegenden \\
\hline Bildungsniveau & Niedrig: ohne abgeschl. & $10,3 \%$ \\
(Gesamtbevölkerung) & Ausbildung & $4,5 \%$ \\
& Hoch: Studium oder & 4,5 \\
& Aufstiegsfortbildung & \\
\hline
\end{tabular}

Doch welche Rahmenbedingungen gelten für pflegende Studierende? Zunächst gelten die Rechte und Leistungen des Pflegezeitgesetzes und des Familienpflegezeitgesetzes nicht. Hochschulen können selbstständig Regelungen 
in Prüfungs- und Einschreibeordnungen treffen, die beispielsweise Fristveränderungen, Beurlaubungen oder ein Recht auf Teilzeitstudium aufgrund von Pflegeaufgaben betreffen (Langer et al. 2011, S. 21). Die Definition von Angehören ist für Studierende ebenso wenig gesetzlich geregelt. Allerdings ist eine Anlehnung an das Pflegezeitgesetz möglich, welches unter dem Begriff „Angehörige“ Großeltern, Eltern, Schwiegereltern, Ehegatten, Lebenspartner, Partner einer eheähnlichen Lebensgemeinschaft, Geschwister, leibliche Kinder, Adoptiv- oder Pflegekinder sowie Kinder des Ehegatten oder Lebenspartners, Schwieger- und Enkelkinder subsumiert.

\subsubsection{Studierverhalten}

Die Vereinbarkeit von Pflege und Studium ist aufgrund der geringen Zahlen und der geringen Sichtbarkeit dieser Studierendengruppe ein bisher wenig beachtetes Thema in Hochschulen (BMBF 2013, S. 10). Im Folgenden werden daher Ergebnisse zur Situation von Pflegenden allgemein und Daten zu Studierenden mit Pflegeaufgaben herangezogen. Pflege ist durch eine hohe zeitliche Verbindlichkeit und Regelmäßigkeit der Verpflichtung und das intime Verhältnis mit der betreuten Person gekennzeichnet (Preuß 2014, 152 f.). Die Pflege von kranken und alten Menschen ist psychisch belastend, da sie häufig Sorge um die zu pflegende Person und einen Prozess der Verabschiedung beinhaltet. In der öffentlichen Wahrnehmung ist Pflege wenig präsent. Ihr fehlt die positive Konnotation, die etwa mit kleinen Kindern einhergeht (Bischofberger et al. 2009, S. 280; Langer et al. 2011, S. 71).

Die genannten Belastungen spiegeln sich in der signifikant schlechteren Gemütsverfassung der pflegenden Studierenden wider. 27 Prozent der Befragten der QUEST-Umfrage geben an, dass sie ihr Studium nur schlecht mit privaten und beruflichen Terminen vereinbaren können. Bei Studierenden ohne Pflegeaufgaben sind es nur 16 Prozent. Die Pflegenden sehen außerdem die Prüfungsdichte und die Anzahl der Lehrveranstaltungen kritischer (Berthold und Leichsenring o. J.a, $21 \mathrm{ff}$.). Analysen unter deutschen Pflegenden unabhängig vom Studierendenstatus zeigen, dass 70 Prozent der Pflegenden ihre Pflegeleistung als äußerst lohnend empfinden und fast 90 Prozent gut mit ihrer Situation umgehen können. Trotz dieser generell positiven Einschätzung berichten 19 Prozent der Pflegenden, ihre Pflegeaufgaben seien zu anspruchsvoll. Für 15 Prozent führt sie zu psychischen Belastungen, 20 Prozent fühlen sich gar in der Rolle als Pflegende oder Pflegender gefangen. Der gesundheitliche Zustand der Pflegenden verschlechtert sich deutlich, wenn die Stundenzahl der Pflege ansteigt (Döhner et al. 2007, S. 94). Wilz et al. (2005, S. 265) zeigen die besonderen Belastungen am Beispiel der Pflege von Demenzkranken auf. Im Vergleich zur Vergleichsgruppe der Personen 
ohne Pflegeaufgaben treten bei diesen Pflegenden besonders Erschöpfung, Gliederschmerzen und Beschwerdedruck häufiger auf. Schließlich wünschen sich fast 60 Prozent der Befragten sich immer oder häufig mehr Unterstützung bei ihrer Aufgabe (Döhner et al. 2007, S. 94). Dies kann auch als Appell an Hochschulen gesehen werden, im Rahmen ihrer Möglichkeiten die Vereinbarkeit von Pflege und Studium zu verbessern.

Daten aus der QUEST-Erhebung zeigen, dass Studierende mit Pflegeaufgaben schlechter sozial integriert sind als Studierende ohne Pflegeverantwortung (Berthold und Leichsenring o. J.a, S. 20). Zur akademischen Integration liegen keine Erkenntnisse vor.

\subsubsection{Anforderungen an das Studium}

Die Anforderungen von Pflegenden an das Studium sind im Gegensatz zu denen von Studierenden mit Kind bisher kaum erforscht (Kunadt et al. 2014, S. 33). Die vorliegenden Artikel weisen jedoch auf zwei Faktoren hin. Ein erster Faktor ist die flexible Gestaltung der Prüfungsordnungen und Veranstaltungen. Um die Auswirkungen zeitlicher und psychischer Belastungen durch plötzlich auftretende oder andauernde Pflegeverantwortung abzumildern, können Hochschulen Auszeiten, die Verlängerung des Studiums oder die Verschiebung von Prüfungen ermöglichen. In formalisierter Art und Weise kann der (vorübergehende) Wechsel in ein Teilzeitstudium den Druck auf die Studierenden reduzieren. Da sich Pflegezeiten mit Lehrveranstaltungen überschneiden können, kann E-Learning die nötige Zeit- und Ortsunabhängigkeit herstellen (Langer et al. 2011, S. 76). Kunadt et al. (2014, S. 32 f.) zeigen, dass die Hochschulen bisher überwiegend Informationsleistungen vorhalten und regionale Anbieter vermitteln. Daher besteht eine Diskrepanz zwischen den Bedarfen der pflegenden Studierenden und den Angeboten der Hochschulen. 
Tabelle 8 Zusammenfassung der Anforderungen von Studierenden mit Pflegeaufgaben an die Studienstrukturen.

\begin{tabular}{|c|c|c|}
\hline Thema & Anforderung & \multirow{14}{*}{$\begin{array}{l}\text { Quellen } \\
\text { Berthold und } \\
\text { Leichsenring o. } \\
\text { J.a, } \\
\text { Langer et al. } \\
\text { (2011), } \\
\text { Kunadt et al. } \\
\text { (2014), } \\
\text { Reichert (2012). }\end{array}$} \\
\hline Ort und Zeit von & Flexibilität, E-Learning, geringe & \\
\hline Lehrveranstaltungen & Anwesenheitspflichten & \\
\hline Umfang der & Hohe Wahlmöglichkeiten & \\
\hline Präsenzlehre und & & \\
\hline Verteilung des & & \\
\hline Arbeitsaufwands & & \\
\hline $\begin{array}{l}\text { Studienunterbrechung } \\
\text { und Studiendauer }\end{array}$ & Urlaubssemester & \\
\hline $\begin{array}{l}\text { Flexibilität des } \\
\text { Studienformats }\end{array}$ & Teilzeitstudium & \\
\hline Beratung und & Beratungsangebote für Pflegende & \\
\hline \multirow[t]{4}{*}{ Betreuung } & inkl. Vermittlung eines & \\
\hline & regionalen Netzwerks, & \\
\hline & Vermittlung von & \\
\hline & Notfallbetreuung & \\
\hline
\end{tabular}

\subsection{Studierende mit Behinderung}

\subsubsection{Lebens- und Studiensituation}

An deutschen Hochschulen haben 23 Prozent der Studierenden eine Beeinträchtigung oder chronische Erkrankung, wobei etwa die Hälfte hiervon im Studium eingeschränkt ist (Middendorff et al. 2017, S. 119). ${ }^{5}$ Behinderung wird je nach beispielsweise psychologischem, sozialwissenschaftlichem oder medizinischem Bezugsrahmen und kulturellem Kontext unterschiedlich definiert. In den letzten Jahrzehnten hat sich die Sichtweise auf Behinderungen gewandelt: von einer Defizitorientierung hin zu einer Entstehung von Behinderung durch Austauschprozesse zwischen Person und Umwelt (Sasse und Moser 2016, 139 ff.). Im Sinne des sozialen Modells besteht das Behinderungskonzept damit aus einer physischen oder psychischen Beeinträchtigung, die im Zusammenspiel mit gesellschaftlichen Verhältnissen zu Behinderungen führt (Hughes und Paterson 1997, S. 327). Wie sich eine Beeinträchtigung auswirkt und wann sie Studierende

\footnotetext{
${ }^{5}$ Auszüge dieses Unterkapitels sind ähnlich im Artikel Buß (2018): Erfolgreich studieren mit Beeinträchtigung durch Interaktionen im Studium. Beiträge zur Hochschulforschung, 40 (3) erschienen.
} 
im Studium behindert, hängt damit auch von den Studienbedingungen und der Hochschulkultur ab (Dobusch et al. 2012, S. 74).

Beeinträchtigungen sind vielfältig und wirken sich unterschiedlich auf das Studium aus. Eine Umfrage des Deutschen Studentenwerks zeichnet ein umfassendes Bild von der Situation Studierender mit Behinderung und chronischer Erkrankung. Die Mehrheit der Studierenden mit Beeinträchtigung leidet unter psychischen und seelischen Erkrankungen. Dies sind beispielsweise Depressionen, Angsterkrankungen, Psychosen, Süchte oder psychosomatische Erkrankungen (Stange 2014, S. 21 ff.). Die nächstgrößere Gruppe sind Personen mit chronisch-somatischen Erkrankungen, gefolgt von Personen mit Teilleistungsstörungen wie etwa Legasthenie, Sehbeeinträchtigungen sowie Bewegungsbeeinträchtigungen. Die kleinste Gruppe bilden Personen mit Hör- und Sprechbeeinträchtigungen. Eine amtlich festgestellte Behinderung oder Schwerbehinderung liegt bei lediglich 13 Prozent der betroffenen Studierenden vor, wobei 58 Prozent nie eine Überprüfung beantragt haben. Insgesamt werden psychische Beeinträchtigungen oder Teilleistungsstörungen seltener amtlich als Behinderung anerkannt. Die amtliche Feststellung ist kein Indiz dafür, wie stark die Studierenden die Auswirkungen ihrer Behinderung auf das Studium wahrnehmen (Unger et al. 2012, S. 21 ff.).

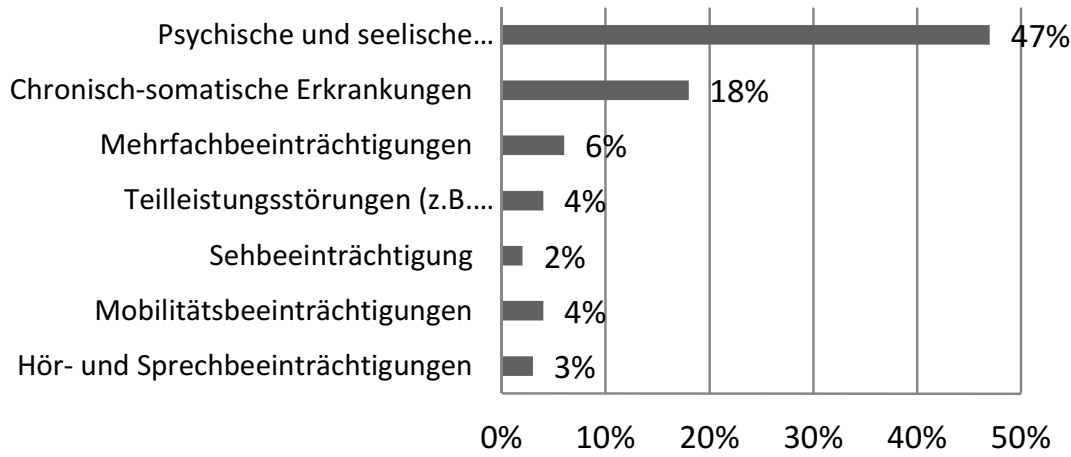

Abbildung 5 Art der Beeinträchtigung. Eigene Darstellung mit Daten aus Middendorff et al. (2017, S. 37)

Anteil und Stärke der Beeinträchtigungen nehmen mit dem Alter der Studierenden zu und treten bei etwa einem Viertel der Studierenden erst während des Studium auf (Middendorff et al. 2013, S. 457; Unger et al. 2012, S. 28). Die 
Beeinträchtigungen sind beim überwiegenden Teil der Studierenden nicht sichtbar. Nur sechs Prozent geben an, dass sie sofort sichtbar sei und bei weiteren $32 \%$ ist dies nach längerem Kontakt der Fall. Die Beeinträchtigung wirkt sich unterschiedlich stark auf das Studium aus. $6 \%$ aller Studierenden werden von einer Beeinträchtigung stark oder sehr stark im Studium behindert. Studierende, die keine Auswirkung auf das Studium berichten, werden daher als Studierende mit Beeinträchtigung definiert. Studierende mit Studieneinschränkung gelten nachfolgend als Studierende mit Behinderung.

\subsubsection{Studierverhalten}

Die Beeinträchtigungen unterscheiden sich abhängig von den Studienbedingungen stark in ihren Auswirkungen auf das Studierverhalten. Da kaum Daten zum Studierverhalten bei spezifischen Beeinträchtigungen vorliegen, fokussieren die nachfolgenden Analysen auf allgemeine Tendenzen. Im Zusammenspiel mit den Studienbedingungen hängen Beeinträchtigungen unter anderem mit Schwierigkeiten bei der Organisation des Studiums, den Lehrveranstaltungen und Prüfungen sowie Studienunterbrechungen durch Krankheitsschübe zusammen. So wird es als Folge von unter anderem Seh- und Hörbehinderungen oder psychischen Beeinträchtigungen schwieriger, konzentriert alle notwendigen Informationen in einer Lehrveranstaltung aufzunehmen. Dies gilt besonders dann, wenn den Studierenden nicht die geeigneten Hilfsmittel zur Verfügung stehen, die Lehrenden nicht auf die Bedürfnisse der beeinträchtigten Studierenden eingehen oder es kaum Regenerationszeiten zwischen den Veranstaltungen gibt (Kerst 2016, S. 149; Meister 1998, S. 100 ff.; Unger et al. 2012, S. 232 ff.). Viele Studierende müssen darüber hinaus ihr Studierverhalten im Laufe des Studiums anpassen. Häufig muten sie sich zu Beginn zu viele Veranstaltungen zu, da sie ähnlich schnell und gut wie Studierende ohne Beeinträchtigung studieren möchten (Meister 1998, S. 105).

Darüber hinaus fühlen sich Studierende mit Beeinträchtigung häufiger exkludiert und isoliert (Meister 1998, S. 66 ff.). Bisher liegen nur wenige Studien zur sozialen und akademischen Integration dieser Studierendengruppe vor. Auch wenn Studierende in Deutschland die Kontaktmöglichkeiten an Hochschulen schätzen, berichten sie von Problemen, wenn die Gesprächspartner unsicher sind oder wenig Erfahrung im Umgang mit Menschen mit Beeinträchtigung haben (Herzig 2015, S. 33; Meister 1998, S. 66 f.). Etwa 20 Prozent der Studierenden haben das Gefühl, dass sich Kommilitonen ihnen gegenüber gleichgültig verhalten (Meister 1998, S. 71). Weiterhin geben Studierende mit Beeinträchtigung eher an, nicht in die Hochschule zu passen (Adams und Proctor 2010, S. 175) oder isoliert $\mathrm{zu}$ sein (Kerst 2016, S. 149). Insgesamt bewerten die Studierenden mit 
Behinderung die soziale Integration deutlich schlechter als Studierende ohne Behinderung (Berthold und Leichsenring o. J.c, S. 19; Buß 2018). Forschungsergebnisse aus den USA weisen auf Unterschiede in der Integration im Studienverlauf hin. Studierende mit und ohne Beeinträchtigung schätzten drei Wochen nach Studienbeginn die soziale Integration im Mittel noch gleich ein (Shepler und Woosley 2012, S. 44). Im späteren Verlauf des Studiums hingegen sind Studierende mit Beeinträchtigung schlechter sozial integriert (Adams und Proctor 2010, S. 175).

Auch wenn 85 Prozent der Studierenden im Sinne der akademischen Integration einen persönlichen Kontakt zu Lehrenden haben, wünschen sie sich von den Lehrenden eine häufigere proaktive Vorgehensweise und eine hohe Verlässlichkeit der Absprachen (Meister 1998, S. 83). Auch den kompetenten und informierten Umgang mit Beeinträchtigungen und Nachteilsausgleichen mahnen Studierende an (Herzig 2015, S. 33; Unger et al. 2012, S. 180). Über diese Kritik am Umgang mit der Beeinträchtigung hinaus bewerten diese Studierenden den Kontakt zu Lehrenden und Mitarbeitenden nur etwas schlechter als Studierende ohne Beeinträchtigung (Adams und Proctor 2010, S. 175; Kerst 2016, S. 147). Dieses Ergebnis weist darauf hin, dass eine Beeinträchtigung besonders im Umgang mit Kommilitonen einschränkend wirkt.

Betrachtet man die Herausforderungen der Zielgruppe differenziert nach Hochschultyp, so zeigen sich einige Unterschiede. Studierende mit Beeinträchtigung, die an kleinen Hochschulen studieren, haben etwas geringere Probleme im Studium als andere. Möglicherweise ist ein Grund hierfür die persönlichere Betreuung. Da Fachhochschulen häufig kleiner sind als Universitäten, würde dies für eine bessere Studiensituation an Fachhochschulen sprechen. Dies ist allerdings pauschal nicht zu bestätigen, da die Studierenden dieses Hochschultyps besonders starke Probleme mit der Prüfungsdichte angeben. Schließlich scheint die im Rahmen der Einführung von Bachelor- und Masterstudiengängen durchgeführte Komprimierung und Strukturierung zu größeren Problemen mit den Leistungsanforderungen zu führen (Unger et al. 2012, S. 155).

\subsubsection{Studienerfolg}

Einige wenige Studien befassen sich mit dem Studienerfolg von Studierenden mit Behinderung. Sie zeigen im Kern, dass Beeinträchtigungen das Risiko eines Studienabbruchs und die Studiendauer erhöhen. Gesundheitliche Probleme spielen bei 10 Prozent der Studienabbrecher eine Rolle; für 4 Prozent sind sie sogar der ausschlaggebende Grund für das Verlassen des Hochschulsystems (Heublein et al. 2010, S. 19). Sind Studierende krank, bewerten sie die Studienbedingungen 
schlechter und den Leistungsdruck höher, was wiederum die Studienabbruchneigung erhöht (Blüthmann et al. 2011, S. 114). Diese Forschungen unterscheiden jedoch nicht zwischen vorrübergehenden Krankheiten und langfristigen Behinderungen. Das Konstanzer Studierendensurvey analysiert Studierende mit Beeinträchtigung und differenziert nach dem Grad der Studienerschwernis. 76 Prozent der Studierenden mit starker Studienerschwernis denken nie an Studienabbruch, wobei es bei Studierenden ohne Beeinträchtigung 90 Prozent sind (Kerst 2016, S. 146). Darüber hinaus studieren Studierende mit Behinderung länger und wechseln häufiger das Studienfach (Middendorff et al. 2013, S. 462). Studierende mit einer (sehr) starken Studienerschwernis benötigen durchschnittlich 8,5 Semester bis zum Studienabschluss; bei der Vergleichsgruppe ohne Beeinträchtigung sind es 6,8 Hochschulsemester (Kerst 2016, S. 145). Trotz der längeren Studiendauer und des höheren Studienabbruchrisikos gibt es Hinweise aus den USA darauf, dass sich Studierenden mit und ohne Behinderung nicht in ihren Noten unterscheiden (Adams und Proctor 2010, S. 176).

\subsubsection{Anforderungen an das Studium}

Die Anforderungen an Studium und Lehre hängen stark von der jeweiligen Beeinträchtigung $\mathrm{ab}$ und können schwer verallgemeinert werden. Nachfolgend seien zwei Beispiele für die unterschiedlichen Auswirkungen dargestellt. Studierende mit Aufmerksamkeits-Defizit-Hyperaktivitäts-Störung (ADHS) haben Schwierigkeiten, sich selbst im Lernprozess zu regulieren und steuern. Coachings zur besseren Selbsteinschätzung, zur realistischen Planung des Studiums und zur Kontrolle des Lernprozesses zeigen signifikante positive Auswirkungen auf die Lernstrategien (Field et al. 2013, S. 74 ff.). Studierende mit Sehbeeinträchtigung sind darauf angewiesen, die Materialien in eine für sie lesbare Form umzusetzen. Je nach Grad der Sehbeeinträchtigung kann dies beispielsweise durch Sehhilfen, Umsetzung in Braille-Schrift oder Vorleseprogramme geschehen. Lehrende können unter anderem darauf achten, dass Unterrichtsmaterialien möglichst früh zur Verfügung gestellt werden und dass Visualisierungen an der Tafel oder in Präsentationen gut lesbar sind (Universität zu Köln 2010, S. 5). Handreichungen zum Umgang mit Beeinträchtigungen bietet die Homepage des Deutschen Studentenwerks.

Die Anforderungen an die Studienstruktur sind im Gegensatz zu den Anforderungen an die Lehre und Nachteilsausgleiche etwas einheitlicher zu erfassen. Die vorliegenden Studien zu diesem Thema weisen insbesondere auf das Thema Flexibilität von Zeit und Organisation des Studiums hin, damit beispielsweise Krankheitsschübe ausgeglichen werden können (Stange 2014, S. 20). Über 60 Prozent der Studierenden haben Probleme mit den zeitlichen 
Vorgaben des Studiums und der starren Organisation des Studiums. Die Probleme differieren zwischen den Beeinträchtigungsarten und steigen bei höherer Studienerschwernis deutlich an (Unger et al. 2012, S. 147 ff.). Einschränkend ist $\mathrm{zu}$ beachten, dass in den vorliegenden Studien überwiegend die Probleme der Zielgruppe benannt werden. Rechtlich können Studierende mit Beeinträchtigungen ihre benötigte Flexibilität durch individuell zu beantragende Nachteilsausgleiche herstellen. Dies sind jedoch immer nur Ausnahmen von der vorgegebenen Strukturregel. Wenn Hochschulen insgesamt auf eine gewisse Flexibilität in der Studienstruktur achten, könnte der Bedarf an Einzelfalllösungen und Ausnahmen reduziert werden. Viele der genannten Anforderungen treffen auch auf Studierende mit Familienpflichten oder Berufstätige zu, so dass deren Umsetzung einer großen Anzahl von Studierenden entgegenkommen würde.

Tabelle 9 Zusammenfassung der Anforderungen von Studierenden mit Beeinträchtigung an die Studienstrukturen.

\begin{tabular}{|c|c|c|}
\hline Thema & Anforderung & \multirow{15}{*}{$\begin{array}{l}\text { Quellen } \\
\text { Unger et al. } \\
\text { (2012), } \\
\text { Stange (2014), } \\
\text { Meister } \\
\text { (1998), } \\
\text { Hildegardis- } \\
\text { Verein e.V. } \\
\text { (2013), } \\
\text { Field et al. } \\
\text { (2013). }\end{array}$} \\
\hline Ort und Zeit von & Wahlmöglichkeiten, flexible & \\
\hline Lehrveranstaltungen & $\begin{array}{l}\text { Abfolge der Module, E-Learning, } \\
\text { Nachteilsausgleiche }\end{array}$ & \\
\hline Umfang der & Moderate Anzahl & \\
\hline Präsenzlehre und & Präsenzveranstaltungen, die & \\
\hline Verteilung des & gleichmäßig verteilt sind und & \\
\hline Arbeitsaufwands & Pausen beinhalten, gleichmäßige & \\
\hline & Verteilung der Prüfungen & \\
\hline Studienunterbrechung & Längere Studiendauer, flexible & \\
\hline und Studiendauer & $\begin{array}{l}\text { Beurlaubungen und Nachholen des } \\
\text { in Krankheitszeiten Versäumten }\end{array}$ & \\
\hline $\begin{array}{l}\text { Flexibilität des } \\
\text { Studienformats }\end{array}$ & Teilzeitstudium & \\
\hline Beratung und & Intensive Begleitung in Mentoring & \\
\hline \multirow[t]{3}{*}{ Betreuung } & oder Coaching, Ruhe- und & \\
\hline & Rückzugsräume, & \\
\hline & Barrierefreiheit der Räume & \\
\hline
\end{tabular}

\subsection{Index der zeitlichen Restriktionen}

In den vorgangegangenen Unterkapiteln wurde die Lebens- und Studiensituation von vier Studierendengruppen analysiert. Um die Belastungen besser vergleichen $\mathrm{zu}$ können, wird nachfolgend die zeitliche Einschränkung der 
Studierendengruppen fokussiert und in einem Index zusammengebracht. Dieser wurde schon in Kapitel 2.2 theoretisch eingeführt. Der Index differenziert vier Kategorien. Studierende, die keinem Beruf nachgehen, keine Sorgeaufgaben haben und keine gesundheitlichen Einschränkungen aufweisen, haben keine zeitlichen Restriktionen (1. Kategorie). Weiterhin gibt es Studierende mit geringen (2. Kategorie), mittleren (3. Kategorie) oder hohen (4. Kategorie) zeitliche Restriktionen. Die Einteilung erfolgt anhand entsprechender Literatur zum Zeitbudget. Die Ergebnisse dieser Forschung sowie der Index sind in Tabelle 10 zusammengefasst.

Tabelle 10 Index zeitlicher Restriktionen.

\begin{tabular}{|c|c|c|c|c|}
\hline & \multicolumn{4}{|c|}{ Zeitliche Restriktionen (Index-Punkte) } \\
\hline $\begin{array}{l}\text { Studierende } \\
\text { mit... }\end{array}$ & Keine (0) & Gering (1) & Mittel (2) & Hoch (3) \\
\hline $\begin{array}{l}\text { Erwerbs- } \\
\text { tätigkeit }\end{array}$ & $\begin{array}{l}\text { Keine } \\
\text { Erwerbstätigkeit }\end{array}$ & 1-9 Stunden & 10-19 Stunden & $\begin{array}{l}\text { Mehr als } \\
19 \\
\text { Stunden }\end{array}$ \\
\hline Kind & $\begin{array}{l}\text { Kein Kind unter } \\
16 \text { Jahren }\end{array}$ & $\begin{array}{l}\text { Kind unter } 16 \\
\text { Jahren, Elternteil } \\
\text { betreut nicht } \\
\text { hauptsächlich } \\
\text { selbst }\end{array}$ & $\begin{array}{l}\text { Kind unter } 16 \\
\text { Jahren, } \\
\text { Elternteil } \\
\text { betreut } \\
\text { hauptsächlich } \\
\text { selbst }\end{array}$ & 1 \\
\hline $\begin{array}{l}\text { Pflege- } \\
\text { aufgaben }\end{array}$ & $\begin{array}{l}\text { Keine } \\
\text { Pflegeaufgaben }\end{array}$ & $\begin{array}{l}\text { Pflegeaufgabe, die } \\
\text { subjektiv nicht } \\
\text { zeitlich im } \\
\text { Studium } \\
\text { einschränkt }\end{array}$ & $\begin{array}{l}\text { Pflegeaufgabe, } \\
\text { die im Studium } \\
\text { einschränkt }\end{array}$ & I \\
\hline Behinderung & $\begin{array}{l}\text { Keine } \\
\text { Behinderung/ } \\
\text { Beeinträchtigung } \\
\text { ohne Studien- } \\
\text { einschränkung }\end{array}$ & $\begin{array}{l}\text { Beeinträchtigung } \\
\text { mit Studien- } \\
\text { einschränkung }\end{array}$ & I & 1 \\
\hline
\end{tabular}

Um die zeitlichen Restriktionen durch Erwerbstätigkeit in die Kategorien einordnen zu können, werden die zuvor aufgezeigten Nachteile durch Berufstätigkeit im Studierverhalten sowie für den Studienerfolg zurate gezogen. Studierende können ihre Berufstätigkeit aufgrund entsprechender Arbeitsverträge oder Stundenabrechnungen relativ präzise angeben, weshalb der Index auf wöchentlichen Arbeitszeiten in der Semesterzeit beruht.

Auch wenn die zitierten Studien keine einheitlichen Zahlen liefern, so wird doch deutlich, dass erst ab einem Umfang von etwa acht bis zehn Stunden geringe 
negative Effekte auf die Studiendauer entstehen können (Body et al. 2014, S. 9; Furr und Elling 2000, S. 461). Einige Studien weisen sogar positive Effekte geringer Berufstätigkeit auf Noten aus (Darolia 2014, S. 44; Sprietsma 2015, S. 12). Aus diesem Grund werden bis zu neun Stunden Berufstätigkeit als geringe zeitliche Restriktion eingestuft. Arbeiten Studierende zehn bis 19 Stunden, sind sie schon etwa zwei volle Tage in der Woche beruflich gebunden. Die Studiendauer verlängert sich bei dieser Erwerbstätigkeit deutlich (Body et al. 2014, S. 9; Brandstätter und Farthofer 2003, S. 140). Daher wird der Index mit einer mittleren Restriktion belegt. Sind Studierende mehr als 20 Stunden berufstätig, entspricht dies mehr als 50 Prozent der regulären Arbeitszeit. Dies reduziert die in das Studium investierte Zeit deutlich stärker als eine Teilzeitberufstätigkeit und hat deutlich negative Auswirkungen auf den Studienerfolg (Pechar und Wroblewski 1998, S. 118). Studierende mit einer wöchentlichen Arbeitszeit von mehr als 20 Stunden unterliegen damit hohen zeitlichen Restriktionen (Triventi 2014, S. 10).

Bei Studierenden mit Kind ist es im Gegensatz zur Berufstätigkeit schwierig, eine konkrete Wochenstundenanzahl zu schätzen. Berechnungen des Zeitbudgets von Studium und Haushaltsarbeit ermöglichen hier eine erste Annäherung. Helfferich et al. (2007, S. 91) analysieren die Belastungen von Studierenden mit Kind und deren Zeitbudgets. Studierende mit Kind geben an, dass sie kaum Zeit für sich selbst oder Erholungsphasen haben. Dieser Effekt verstärkt sich durch berufliche Verpflichtungen. Mehrere Studien beschäftigen sich mit der Zeitaufwendung von Studierenden mit Kind. Je nach Erhebungsmethode und betrachteten Fächern kommen sie zu unterschiedlichen Ergebnissen. Nach Daten aus Baden-Württemberg wenden Mütter 26 Stunden und Studierende ohne Kind 34 Stunden pro Woche für das Studium auf. Bei Männern zeigen sich mit jeweils 34 Stunden pro Woche keine Unterschiede im Studierverhalten (Helfferich et al. 2007, S. 93). Studierende der Universität Bochum studieren durchschnittlich 23 Stunden, wobei Mütter 15 und Väter 19 Stunden für das Studium aufwenden. Die geringere Zeit für das Studium ist durch Haushaltstätigkeiten und ggf. Kinderbetreuung begründet, die bei Müttern 22 Stunden, bei Vätern zehn Stunden und bei Studierenden ohne Kind nur sechs Stunden ausmachen (Bochumer Studierendenbefragung 2002, zitiert nach BMFSFJ 2004, S. 73). Die Unterschiede sind damit überwiegend auf die durchschnittlich stärkere Belastung von Frauen für die Kinderbetreuung und unbezahlte (Haushalts-)Arbeit zurückzuführen. So unterscheidet sich die Belastung für unbezahlte Arbeit zwischen Männern (2:44 Stunden/Tag) und Frauen (4:07 Stunden/Tag) grundsätzlich. Dies verstärkt sich, sobald ein Kind mit im Haushalt lebt (Gille und Marbach 2004, S. 96). In diesem Fall sind Frauen durchschnittlich etwa zweieinhalb Stunden und Männer nur etwa eine halbe Stunde zusätzlich pro Tag im Haushalt aktiv. Aus den Zeitinvestitionen 
ergibt sich, dass Frauen häufiger als Männer für die Kinderbetreuung zuständig sind. Die Zuständigkeit für Kinderbetreuung beeinflusst damit die zeitliche Restriktion von Eltern.

Der Index für zeitliche Restriktionen berücksichtigt insbesondere die Zuständigkeit für die Kinderbetreuung. Hierdurch kann indirekt das Geschlecht berücksichtigt werden, da Männer seltener die Hauptverantwortung für die Betreuung tragen. Haben Studierende die Hauptverantwortung für das Kind, so wird im Index eine mittlere Restriktion (zwischen 10 und 20 Stunden pro Woche) vermerkt. Liegt die Verantwortung beim Partner oder bei der Familie, wird eine geringe zeitliche Restriktion von bis zu 10 Stunden in der Woche zugrunde gelegt. Die Belastung durch Kinderbetreuung ist vom Alter und der Selbstständigkeit der Kinder abhängig (Freier 2007, S. 12): Jüngere Kinder sind deutlich betreuungsintensiver als ältere Kinder. Daher ist für den Index festzulegen, ab wann die Kinder so selbstständig sind, dass das Studium der Eltern nicht eingeschränkt wird. Diese Grenze liegt im Folgenden bei 16 Jahren, da für Jugendliche ab diesem Alter die Vollzeitschulpflicht endet. Der Index berücksichtigt daher nur Studierende, deren jüngstes Kind unter 16 Jahre alt ist.

Die Belastung durch Pflegeaufgaben ist schwer zu beziffern, insbesondere da keine Daten zum zeitlichen Umfang der Pflege von Studierenden in Deutschland vorliegen. Das Deutsche Zentrum für Altersfragen hat die Pflegeleistungen von für 40- bis 85-jährige Angehörige untersucht; diese werden nachfolgend als Annäherung an die zeitlichen Restriktionen durch Pflegeaufgaben genutzt. Zunächst zeigen sich deutliche Unterschiede zwischen erwerbstätigen und nicht erwerbstätigen Pflegenden. Gehen die Pflegenden einem Beruf nach, so leisten sie durchschnittlich 16 Stunden Pflege pro Woche. Sind sie nicht erwerbstätig, sind es hingegen 26 Stunden (Klaus und Tesch-Römer 2014, S. 7). Frauen pflegen darüber hinaus mehr als doppelt so häufig wie Männer und leisten darüber hinaus mehr Stunden. Das Bildungsniveau hat einen deutlichen Effekt auf das Engagement in der Pflege, da Menschen mit mittlerem oder hohem Bildungsabschluss mit 15 Stunden nur halb so viele Stunden pflegen wie Menschen mit niedrigem Abschluss. Da die Studierenden einen mittleren (Ausbildung oder höherer Bildungsabschluss) oder hohen Bildungsabschluss (Studium oder Aufstiegsfortbildung) aufweisen und ähnlich wie Erwerbstätige Verpflichtungen durch das Studium haben, ist ein Stundenumfang von etwa 15 Stunden anzunehmen. Langer et al. (2011, S. 46) weisen darauf hin, dass die zeitliche Belastung der pflegenden Studierenden vom Bedarf der zu pflegenden Person abhängt. Daher können die zeitlichen Restriktionen unter oder über dem Durchschnitt von 15 Stunden liegen. Um dieser Situation Rechnung zu tragen, dient die subjektive Einschätzung der Einschränkungen im Studium durch die Pflegeaufgabe als weitere Annäherung an die zeitliche Restriktion. Wenn 
Studierende pflegen, sich aber subjektiv nicht im Studium eingeschränkt fühlen, haben sie eine geringe zeitliche Restriktion. Eine mittlere zeitliche Restriktion liegt vor, wenn sich die pflegenden Studierenden in ihrem Studium zeitlich eingeschränkt fühlen.

Die Beeinträchtigung bedeutet für 59 Prozent der beeinträchtigten Studierenden eine (sehr) starke Studienerschwernis. Diese Studienerschwernis führt in dem meisten Fällen zu einem höheren zeitlichen Bedarf für die Organisation des Privatlebens und des Studiums. Studierende mit Beeinträchtigung müssen ungleich mehr Kraft und Energie aufbringen, um die gleichen Aktivitäten durchzuführen. Sie benötigen darüber hinaus mehr Ruhepausen, um in der Lehrveranstaltung oder im Selbststudium konzentriert bleiben zu können (Holm-Hadulla et al. 2009, S. 352; Meister 1998, S. 93 ff.). Sie leiden besonders häufig unter Konzentrationsschwierigkeiten und Ängsten (HolmHadulla et al. 2009, S. 352 ff.). Im Fall von Studierenden mit Lernbeeinträchtigungen ist außerdem eine geringere Lesegeschwindigkeit nachgewiesen (Lewandowski et al. 2016, S. 572). Sind Studierende in der Motorik oder der Seh- und Hörfähigkeit stark eingeschränkt, so sind sie häufig von individueller Unterstützung für ihre Fortbewegung oder Übersetzung durch Gebärdendolmetscher abhängig. Ist eine solche individuelle Unterstützung nicht in ausreichendem Umfang oder nicht zuverlässig verfügbar, kostet dies den Studierenden viel Zeit oder macht gar eine Teilnahme an Veranstaltungen unmöglich (Meister 1998, S. 93 ff.; Unger et al. 2012, S. 135 ff.). Aufgrund der hohen Diversität unter den Studierenden mit Behinderung ist eine konkrete Schätzung der zeitlichen Belastung durch die Beeinträchtigung schwierig. Analysen zum Zeitbudget zeigen, dass Studierende mit studieneinschränkender Beeinträchtigung ähnlich viel Zeit in das Studium investieren wie Studierende ohne Beeinträchtigung. Die Zeit für das Studium reduziert sich allerdings, wenn die Beeinträchtigung das Studium (sehr) stark erschwert. Die Verteilung der Zeit differiert leicht, da Studierende mit Behinderung mehr Zeit für das Selbststudium verwenden und weniger Zeit für Lehrveranstaltungen (Meister 1998, S. 96 ff.; Middendorff et al. 2013, S. 473). Die Ergebnisse zeigen, dass die Studierenden mit Beeinträchtigungen durchaus zeitlich eingeschränkt sind. Da sie nicht deutlich weniger Zeit in das Studium investieren als Studierende ohne Beeinträchtigung, ist daher durchschnittlich eine geringe zeitliche Restriktion anzunehmen. Diese kann allerdings im Einzelfall deutlich höher ausfallen, was allerdings in der Skala mit den vorliegenden Daten nicht abgebildet werden kann. In der Skala wird daher eine geringe Restriktion für Studierende mit Studieneinschränkung angenommen.

Sind die Studierenden aus mehreren Gründen zeitlich eingeschränkt, zum Beispiel durch Erwerbstätigkeit und Beeinträchtigungen, werden diese Restriktionen addiert. Die Studierenden erreichen einen entsprechend höheren 
Wert in der Gesamtskala. Zur konkreten Berechnung des Index anhand der befragten Studierenden sei auf das Kapitel 7.1 verwiesen.

Open Access Dieses Kapitel wird unter der Creative Commons Namensnennung 4.0 International Lizenz (http://creativecommons.org/licenses/by/4.0/deed.de) veröffentlicht, welche die Nutzung, Vervielfältigung, Bearbeitung, Verbreitung und Wiedergabe in jeglichem Medium und Format erlaubt, sofern Sie den/die ursprünglichen Autor(en) und die Quelle ordnungsgemäß nennen, einen Link zur Creative Commons Lizenz beifügen und angeben, ob Änderungen vorgenommen wurden.

Die in diesem Kapitel enthaltenen Bilder und sonstiges Drittmaterial unterliegen ebenfalls der genannten Creative Commons Lizenz, sofern sich aus der Abbildungslegende nichts anderes ergibt. Sofern das betreffende Material nicht unter der genannten Creative Commons Lizenz steht und die betreffende Handlung nicht nach gesetzlichen Vorschriften erlaubt ist, ist für die oben aufgeführten Weiterverwendungen des Materials die Einwilligung des jeweiligen Rechteinhabers einzuholen.

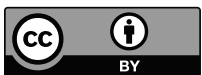

